\title{
NONDESTRUCTIVE TESTING SYSTEM FOR EGGSHELLS BASED ON DSP
}

\author{
Lihong $\mathrm{He}^{1}$, Dejun Jiang ${ }^{1}$, Lan Liu ${ }^{1}$, Jingang Liu ${ }^{2, *}$ \\ ${ }^{1}$ Department of Mechanical and Electrical Engineering, Hunan Institute of Engineering, \\ Xiangtan, China, 411104 \\ ${ }^{2}$ Department of mechanical and automotive engineering, Hunan University, Changsha, \\ China, 410082 \\ * Corresponding author, Address: Room 533, 17th Dormitory, Hunan University, Changsha, \\ 410082, P. R. China, Tel: +86-731-2855111,Fax:+86-732-2855114,Email: wellbuild@126.com
}

Abstract: In order to check and eliminate the cracked eggs quickly and exactly in the process of egg products, a nondestructive testing system for eggshells based on digital signal processor (DSP) was established. The system utilized a TMS320VC33 DSP as a hardware platform, and has many functions such as controlling knock-equipment, sampling and processing the acoustics signals, judging the cracked-shell eggs, communicating with the host computer etc. In order to improve the detecting accuracy, 6 characteristic parameters were extracted in cepstrum domain besides the common used characteristic parameters in frequency domain as candidate distinguishing factors. Step discriminant was used to optimize the combination of these characteristic parameters, then established the optimum discriminant functions of normal eggshells and cracked eggshells respectively according to the step discriminant result. The accurate recognition ratio for cracked eggs is up to 95.5 percent, and the system can check 12 eggs per sec, which satisfied the requirements of the process of egg products.

Keywords: nondestructive testing, acoustic signal, cepstrum, power spectrum

\section{INTRODUCTION}

In egg processing plants, culling the cracked-shell eggs is an important working procedure, which is largely done by handwork currently. However, the quality of eggs detected by handwork cannot be guaranteed due to variable detection accuracy that results from difference in workers'

He, L., Jiang, D., Liu, L. and Liu, J., 2008, in IFIP International Federation for Information Processing, Volume 259; Computer and Computing Technologies in Agriculture, Vol. 2; Daoliang Li; (Boston: Springer), pp. 1147-1154. 
experience, emotion and physical capability. Furthermore, the workers' working conditions and labor intension is too bad. So it is very urgent to find a scientific, applicable and fast detecting method for eliminating cracked-eggs.

At present, there are two different automatic techniques employed in quality detection and sorting of eggs: machine vision and image analysis; and mechanical stiffness measurements. The accuracy of the machine vision method relies on the resolution of the camera, the sorting algorithm and the type of defect. Machine vision inspection works excellently for dirty shells, broken shells and odd shapes, however, detection of small cracks is more difficult (Goodrum et al., 1992; Patel et al., 1998; Nakano et al., 2000; Garcia-Alegre et al., 2000). The mechanical stiffness measurements were based on the measurement and analysis of the mechanical behavior of the eggshell (Nakano et al., 2001). Ketelare et al. developed a method that eggshell crack detection was based on the analysis of the acoustically measured frequency response of an egg excited with a light mechanical impact on different locations on the eggshell equator. This method allowed a crack detection level of $90 \%$ and a false reject level of less than $0.5 \%$ (Ketelare et al., 2000). J. Wang et al. also developed an experimental system to generate the impact force, measure the response wave signal and analyze the frequency spectrum for physical property detection of eggshell (Wang et al., 2004).

In recent years, acoustic testing technique has been developed and seemed to be a promising method in some nondestructive testing system (Duprat et al., 1997; Cho et al., 2000). The same concept was applied herein to the case of detecting and sorting the eggs. In this paper we established an experimental nondestructive testing system for eggshells based on DSP. In the system, acoustic signals of normal eggs and cracked eggs were collected, followed by analysis of the differences between the two groups in power spectrum and cepstrum. The developed hardware platform and the introduction of cepstrum in identification process improved the detection accuracy, ratio, reliability and convenience effectively.

\section{EXPERIMENTAL EQUIPMENTON}

The nondestructive testing system for eggshells was designed with a DSP to enhance the detection rate and accuracy. The developed experimental hardware platform should have many functions such as controlling knockequipment, sampling and disposaling the acoustics signals, identifying the cracked-shell eggs, communicating with the PC etc. In order to achieve the above-mentioned performances, The hardware was designed as several subsystems: electromotor-drive sub-system, the signal-amplify and filter sub- 
system, the signals' starting point identification sub-system, the A/D convertor sub-system, the CPLD logic-control sub-system, the power source sub-system, the main processor sub-system, and the interface sub-system which communicated with the PC etc. The systematic structure figure is shown in Fig. 1.

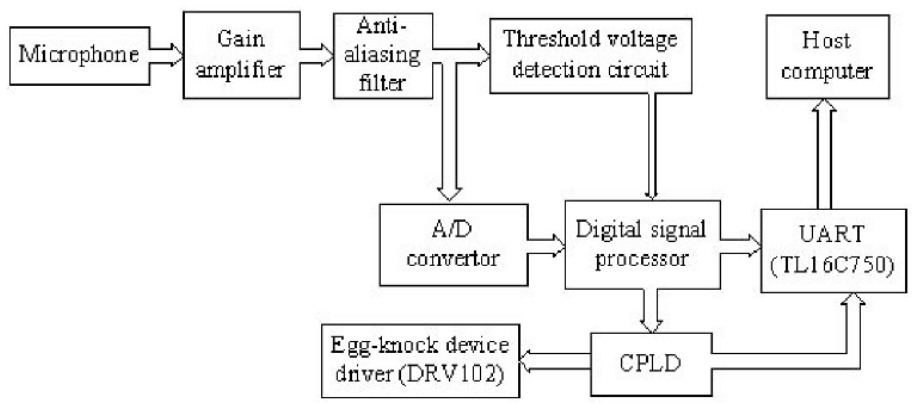

Fig. 1. Schematic diagram of the experimental equipment

Egg-knock device is composed of a solenoid with a return spring. A rubber hammer joints with the armature of the solenoid. The rubber hammer will knock the eggshells when drive current flows through the solenoid coil, and will return rapidly when the drive current is shut off. In order to prevent the rubber hammer break the eggshell the drive current should be shut off at once controlling by the relative program when the eggshell makes a sound.

Since the detecting system must deal with vast floating-point operation and should have high real time performance, TMS320VC33 was selected as the main processor, which performs great efficiency, high speed, low cost and easy exploiture. It is the most perfect DSP in the occasion of floatingpoint operation. The system doesn't need expand the external memory because it has an additional $1 \mathrm{M}$ bits of on-chip SRAM, which is sufficient for detecting calculation. FUM9750BP was selected as the acoustic sensor of the system, which is the single-point electrets microphone whose sensitivity is $-51 \pm 3 \mathrm{~dB}$; frequency response is $100-16,000 \mathrm{~Hz}$. INA217 is the low noises and low distortion audio frequency preamplifier which magnified the lowamplitude signals. A two-order butterworth low pass filter was designed with an operation amplifier OPA134 to filter random noise and baseline drift signals output by the microphone in this work. The cutoff frequency is $10,000 \mathrm{~Hz}$ according to the effective bandwidth of the acoustic signal made by the eggshell (Wen et al., 2002).

In order to reduce the calculation amount of signal processing and realize real time process, the system adopted the threshold circle to check the signals' starting point, which successfully resolve the contradiction between DSP interior memorizer is finite and extracting effective signal by software must use a lot of memory resources. When the objective signal is larger than the threshold voltage, the high speedy comparator TL714 will output low 
level voltage, and then the system considers that the effective signal is coming, and the sampling process is activated at once. The system used the component ADS7813 as the analog-to-digital convertor, which is a 16-Bit $20 u$ s A/D converter with an input multiplexer, sample/hold, clock, reference, and serial data interface. As the maximum convertion time of the convertor is $20 u$ s, the convertion time is sufficient for this detecting system (note that the sampling frequency is $22000 \mathrm{~Hz}$ ). Since both the convertor and the DSP have timing compatible serial data interface, their communication interface is easily to design.

\section{MATIERIALS AND METHOD}

Fresh eggs including cracked eggs were supplied by Hubei Xiantao Food Corporation in China. The extent of eggshell crack in the eggs was varied from broken shells to small cracks. In this study, the rubber hammer knocked once for each eggshell controlled by a program running on DSP. The audio signal made by the eggshell was amplified; filtered and then sampled by the signal gathering program. It was found that the sampling frequency of $22000 \mathrm{~Hz}$ was optimal for accurate sampling after comparing the results obtained under different sampling number, and that the effective signal lasted no more than $4 m \mathrm{~s}$, so 128 point data were sampled for once knocking. In order to find obvious characteristic parameters the digital signal processing program transformed the sampled signal into frequency domain and cepstrum domain. Power spectrum and cepstrum profiles of normal eggs and cracked eggs were compared, from which several characteristic parameters can be retrieved for eggshell crack detection. At last, step discriminant was used to optimize the combination of these characteristic parameters, then establish the optimum distinguishing functions of normal eggshells and cracked eggshells respectively according to the step discriminant result.

\section{EXTRACTION OF OBVIOUS CHARACTERISTIC PARAMETERS}

\subsection{The power spectrum characteristic parameters}

When the rubber hammer knocked the eggshell the eggshell made a sound responding to the pulse excitation. The power spectrum can straightly reflect the acoustic signals' energy distribution in the frequency domain. The power 
spectrum was gained by 128-point Fast Fourier Transform (FFT) algorithm method in this work. According to the property of Discrete Fourier Transformation (DFT), the power spectrum of the acoustic signals is even symmetry on the 64 point; therefore, the paper only analyzed the former 64 point of it. The power spectrum of the typically normal egg's acoustic signal and the typically cracked egg's acoustic signals were shown in Fig. 2. The xcoordinate is the signals' sampling point and the y-axis is the amplitude of the sampling point. Six obvious characteristic parameters were extracted from frequency domain, they were: the first and second peak value in the high frequency band $x_{1}$ and $x_{2}$; the area of power spectrum $x_{3}$; the energy ratio of the low frequency band $x_{4}$; the energy ratio of the low frequency band $x_{5}$; the difference between the energy ratio of the low frequency band and the medium frequency band $x_{6}$. When the egg was knocked the force maybe uneven, in order to eliminate the influence on the detecting accuracy caused by the uneven knocking force, we chose the energy ratio but not the energy as the characteristic parameter. The definitions of the parameter were shown in Table 1, in every formula, $p_{i}$ was the amplitude of the power spectrum, and Peak $k_{k}$ was the value of the $k$ th power spectrum peak in the high frequency band.

Table 1. Definitions of each characteristic parameter form frequency domain

\begin{tabular}{cccccc}
\hline$x_{1}$ & $x_{2}$ & $x_{3}$ & $x_{4}$ & $x_{5}$ & $x_{6}$ \\
\hline $\max p_{i}$ & $\max$ Peak $_{k}$ & $\sum^{63} p_{i}$ & $\sum_{i=7}^{15} p_{i}$ & $\sum_{i=36}^{44} p_{i}$ & $x_{4}-x_{5}$ \\
$33 \leq i \leq 63$ & $k \neq 1$ & $x_{3}$ & & \\
\hline
\end{tabular}

\subsection{The cepstrum characteristic parameters}

When the eggshell is knocked, it should make a sound; the acoustics theory thinks that the acoustic signals are the eggshell's response to the excitation signal $u(n)$, and which can be considered as the outputs of a linear system $h(n)$ excited by the excitation signal $u(n)$. Determined by the eggshell's structure, the linear system $h(n)$ apparently contains the more information of the eggshell.

The theoretical analyses shows that the cepstrum of the acoustic signals $\hat{c}(n)$ is the sum of the cepstrum of the eggshell's impulse response sequence $\hat{h}(n)$ and the cepstrum of the excitation signal $\hat{u}(n)$. The cepstrum of the eggshell's impulse response sequence $\hat{h}(n)$ is a two sided sequence and its amplitude attenuated rapidly when the sampling point $n$ increases. The cepstrum of the excitation signal $\hat{u}(n)$ is a periodic sequence and its amplitude is not zero only when $n \neq k N_{P}$, but at the other point its amplitude is zero. Thus it is highly effectual that the cepstrum of the eggshell's impulse 
response sequence $\hat{h}(n)$ is extracted using the short time-window. The cepstrum of the acoustic signals is shown in Fig. 3. The x-coordinate is the signals' sampling point in the cepstrum domain and the y-axis is the amplitude of the sampling point.The cepstrum figure was weighed by the constant-coefficient 128 and the origin value wasn't included because it was so great that its display will hide other datum. Because the energy of the cepstrum $\hat{h}(n)$ centralized near the origin, the former six cepstrum values including the origin were retrieved as six characteristic parameters in cepstrum domain:

$$
x_{i+7}=\hat{c}(i),(i=0,1, \ldots, 5)
$$

The above mentioned characteristic parameters $x_{1}-x_{12}$ were selected as candidates distinguish factors for differentiating normal eggs and cracked eggs.

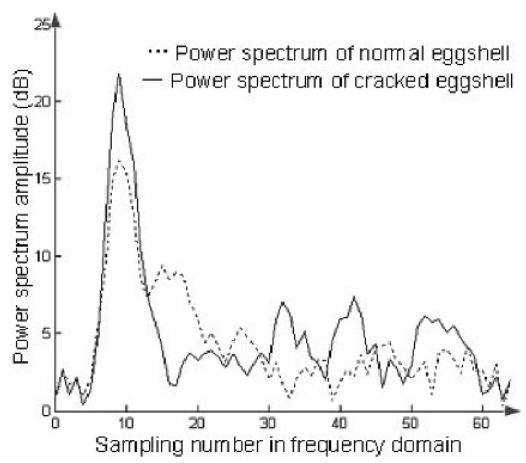

Fig. 2. Power spectral density of the egg

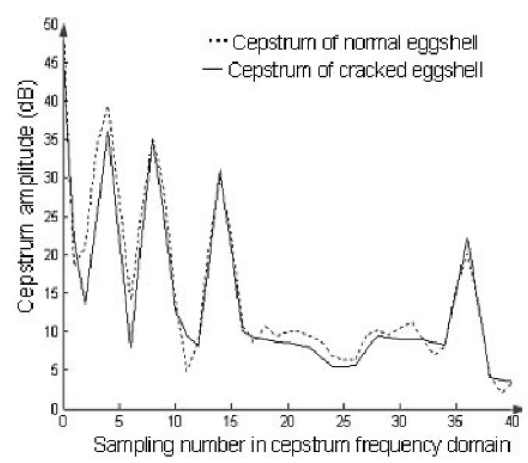

Fig. 3. Cepstrum of the sound given by an egg

\section{STEPWISE DISCRIMINANT ANALYSIS OF EGGSHELL CRACK DETECTION}

Twelve characteristic parameters had been extracted as candidate distinguishing factors. However, some characteristic parameters may be a nonsignificant or reverse influence on the discriminant accuracy, in this study the step-by-step discriminatory method was adopted. The stepwise process of disriminant analysis consisted of identifying the optimal subset of features, as well as the optimal linear discriminating functions of this subset that minimized the pooled expected risk of misclassification (PERM).

Experiments were carried out according to the methods stated in the previous part and a set of data from 200 normal eggs and 150 cracked eggs were collected. However, the data were too large to be listed here. The 
differentiation models of normal eggs and cracked eggs using SAS were developed. Results from the SAS outputs were shown in Table 2.

Table 2. Course STEPDISC

\begin{tabular}{cccccc}
\hline Step & Entered & Removed & $\begin{array}{c}\text { Partial } \\
\text { R-square }\end{array}$ & F Value & Pr $>\mathrm{F}$ \\
\hline 1 & $x_{11}$ & - & 0.6780 & 136.84 & $<.0001$ \\
2 & $x_{4}$ & - & 0.3165 & 33.94 & $<.0001$ \\
3 & $x_{10}$ & - & 0.0739 & 5.03 & 0.0285 \\
4 & $x_{12}$ & - & 0.1111 & 7.75 & 0.0071 \\
5 & $x_{6}$ & - & 0.0935 & 6.29 & 0.0148 \\
6 & $x_{5}$ & - & 0.0309 & 1.91 & 0.1716 \\
7 & & $x_{5}$ & 0.0309 & 1.91 & 0.1716 \\
\hline
\end{tabular}

Differentiation function of cracked eggs is as follows:

$$
\begin{aligned}
G_{z h}= & -81.9501+2.84802 x_{4}-1.86661 x_{6} \\
& +3.77679 x_{10}+0.36412 x_{11}+6.88054 x_{12}
\end{aligned}
$$

Differentiation function of normal eggs is as follows:

$$
\begin{aligned}
G_{z p}= & -103.48+2.11697 x_{4}-1.42783 x_{6} \\
& +0.30921 x_{10}+1.75619 x_{11}+10.6156 x_{12}
\end{aligned}
$$

To further evaluate the preciseness of the models, detection was carried out in a much larger sample in which 1120 normal eggs and 980 cracked eggs were included. Detection data were generated and collected upon execution of the differentiation system. Among the 1120 normal eggs, 64 were wrongly identified as cracked eggs; so the average detection accuracy of the good egg model was $94.2 \%$. On the other hand, among the 980 cracked eggs, 44 eggs were wrongly identified as good eggs; so the average detection accuracy of the cracked egg model was $95.5 \%$.

\section{CONCLUSIONS}

In this study, a nondestructive testing system for eggshells based on digital signal processor (TMS320VC33) is established. Some obvious characteristic parameters extracted from frequency domain and from cepstrum domain were utilized to distinguish the eggshell status. The accurate recognition ratio for cracked eggs of this system is up to 95.5 percent, and the system can check 12 eggs per sec, which satisfied the requirements of the process of egg products.

The discrimination errors for cracked eggs arise mainly from the fact that not all the impact can happen just at or quite near the cracking spot by the egg-knock device. The further experimental results show that the accuracy 
can be improved by increasing the times of impact or detecting different spots by rotating the egg. But other issues may also come up together with the time increase in impact, such as elongation of the data processing period and subsequent reduction in efficiency of the system.

\section{ACKNOWLEDGEMENTS}

This study has been funded by the Department of Science of Huan Institute of Engineering (project number: 120641). The author also thanks Prof. Youxian Wen in Huazhong Agriculture University for introducing active modulation to us and for providing much valuable guidance.

\section{REFERENCES}

Goodrum J W, Elaster R T. Machine vision for crack detection in rotating eggs. Transaction of the ASAE, 1992, 35: 1323-1328

Patel V C, McClendon R W, Goodrum J W. Color computer vision and artificial neural networks for the detection of defects in poultry eggs. Artificial Intelligence Review, 1998, 12: $163-176$

Nakano K, Sasaoka K, Ohtsuka Y. A study on nondestructive detection of abnormal eggs by using image processing. Asian Federation for Information Technology in Agriculture, 2000: 345-352

Garcia-Alegre M C, Ribeiro A, Guinea D, et al. Eggshell defects detection based on color processing. SPIE 2000 Electronic Imaging Conference, 2000

Duprat F, Grotte M, Pietri E, et al. The acoustic impulse response method for measuring the overall firmness of fruit. Journal of Agricultural Engineering Research, 1997, 66: 251-259

Wang J, Jiang R J, Yu Y. Relationship between dynamic resonance frequency and egg physical properties. Food Research International, 2004, 37: 289-294

Ketelaere B D, Coucke P, Baerdemaeker J D. Eggshell crack detection based on acoustic resonance frequency analysis. Journal of Agricultural Engineering Research, 2000, 76: $157-163$

Wen Y, Wang Q, Zong W, et al. Study on crack detection of duck eggs [J]. Huazhong Agricultural University Transaction, 2002, 26: 285-287 (in Chinese)

Cho H K, Choi W K, Paek J H. Detection of surface cracks in shell eggs by acoustic impulse method. Transactions of ASAE, 2000, 43: 1921-1926

Nakano K, Usui Y, Motonaga Y, et al. Development of non-destructive detector for abnormal eggs. Workshop on Control Applications in Post-Harvest and Processing Technology, 2001: 71-76 